\title{
Tris[2-(Acryloyloxy)Ethyl]Isocyanurate Cross-Linked Polyethylenimine Enhanced Exon-Skipping of Antisense 2'-Omethyl Phosphorothioate Oligonucleotide in vitro and in vivo
}

\author{
Mingxing Wang*, Bo Wu, Jason D Tucker, Peijuan Lu and Qilong Lu*
}

Department of Neurology, McColl Lockwood Laboratory for Muscular Dystrophy Research, Cannon Research Center, Carolinas Medical Center, 1000 Blythe Blvd. Charlotte, NC 28231, USA

\begin{abstract}
Branched poly(ester amine)s (PEAs) based on tris[2-(acryloyloxy)ethyl]isocyanurate (TAEI) cross-linked low molecular weight polyethylenimine (LPEI, Mw: $0.8 \mathrm{k} / 1.2 \mathrm{k} / 2.0 \mathrm{k}$ ) polymers have been evaluated for their ability to deliver antisense 2'-O-methyl phosphorothioate RNA (2'-OMePS) through in vitro and in dystrophic mdx mice. Almost all PEAs improved exon-skipping of 2'-OMePS both in vitro and in vivo. Especially the PEAs composed of PEI 2.0k (C series) enhanced 2'-OMePS delivery more effectively than PEI $0.8 \mathrm{k}$ or $1.2 \mathrm{k}$-based series using a GFP reporter-based C2C12 myoblast culture system and in $m d x$ mice. The highest efficiency of targeted exon-skipping of 2'-OMePS was obtained by PEA C14 [TAEI-PEI 2.0k (1:4)] with up to 6 fold increase compared with 2'-OMePS only in mdx mice via intramuscular injection without increased muscle damage. Enhanced exon-skipping and lower toxicity highlight the potential of this series biodegradable PEAs as safe and efficient 2'-OMePS delivery vectors for treating diseases such as muscular dystrophy with splicing modulation.
\end{abstract}

Keywords: Antisense delivery; Exon-skipping; 2'-O-methyl phosphorothioate RNA; Polyethylenimine modification; Muscular dystrophy

\section{Introduction}

With the development of antisense oligonucleotide $(\mathrm{AO})$ chemistry, AO-based exon-skipping has been demonstrated as one of the most promising therapeutic approaches for Duchenne Muscular Dystrophy (DMD) in vitro and in animal model, as well as clinical trials [1-4]. $\mathrm{DMD}$, an incurable, $\mathrm{X}$-linked recessive condition caused by mutations in the dystrophin gene, is the most common and serious form of childhood muscle wasting disease. DMD has an incidence of about 1 in 3500 live male births, and usually leads to death from respiratory or cardiac failure around 30 years old [5,6]. Among the developed antisense oligonucleotide chemistries, neutral charged morpholino oligonucleotide (PMO) [7-16], and 2'-O-methyl-phosphorothioate RNA (2'-OMePS) [17-23] demonstrate more advantages than other counterparts because of their high nuclease resistance and affinity for target sequences. However, clinical trials have revealed limited clinical benefit and failed to halt disease progression. This has been correlated to the inadequate dystrophin induction by the AOs, almost certainly due to narrow window of dosage in the trials. It has been established in a number of animal models that dystrophin levels are well correlated with the functional outcome of muscle, and significant therapeutic value can only be achieved with high dose antisense oligomer regimes, likely considerably higher than the doses being used in ongoing clinical trials. However, long-term treatment with high dose is cost-inhibitive and more important could pose risk of toxicity $[1,2,4,11,13,16,23]$. Furthermore, despite the fact that $2^{\prime}$-OMePS oligomers are resistant to nuclease degradation and stable in biological systems via the phosphorothioate backbone, only a small proportion of 2'-OMePS delivered systemically is taken up into the targeted muscle cells [17-23]. These facts suggest that improving delivery efficiency will be the key for enhancing therapeutic value with the 2 '-OMePS chemistry.

To increase delivery efficiency of negatively charged antisense oligomers 2'-OMePS, polyethylenimine (PEI)-based modifications have been studied as delivery carrier and shown some beneficial effect due to their buffering capacity at virtually any $\mathrm{pH}$. Amongst them, the low molecular weight PEI (LPEI) constructed modifications have validated promise as antisense oligomer delivery vehicles probably related to their molecular size and optimal hydrophilic-lipophilic balance (HLB) and low cytotoxicity [24-27]. Combination of LPEI $2 \mathrm{k}$ with PEG550 copolymer has been demonstrated with enhanced exonskipping in skeletal muscle, but the improvement remains limited, probably due to hydrophilic properties of the polymer, which restricts the interactions between the polymer and 2'-OMePS or the complex and cell membranes [24,25]. We have previously investigated LPEI $(0.8 \mathrm{k} / 1.2 \mathrm{k})$-conjugated pluronics $(2 \mathrm{k}-5 \mathrm{kDa})$ polycarbamates $(\mathrm{PCMs})$, and showed that those composed of moderate size and intermediate HLB (7-23) of pluronics enhanced exon-skipping using 2'-OMePS and PMO with low toxicity as compared with Lipofectamine-2000 (LF-2k) in vitro or PEI 25k in $m d x$ mice [26,27].

We have recently developed a series of novel amphiphilic cationic polymers, branched poly(ester amine)s (PEAs) composed of tris[2(acryloyloxy)ethyl]isocyanurate (TAEI) and low molecular weight polyethylenimine (LPEI, Mw: 0.8k/1.2k/2.0k). The PEAs demonstrated dramatic effects on enhancing the delivery of plasmid transgene and

*Corresponding authors: Mingxing Wang, McColl Lockwood Laboratory for Muscular Dystrophy Research, Neuromuscular/ALS Center, Carolinas Medical Center, 1000 Blythe Blvd. Charlotte, NC 28231, USA, Tel: 1-7043555588; Fax: 1-704-355-1679; E-mail: mingxing.wang@carolinashealthcare.org

Qilong Lu, McColl Lockwood Laboratory for Muscular Dystrophy Research Neuromuscular/ALS Center, Carolinas Medical Center, 1000 Blythe Blvd. Charlotte, NC 28231, USA, Tel: 1-7043551701; Fax: 1-704-355-1679; E-mail: qi.lu@carolinashealthcare.org

Received December 02, 2014; Accepted January 08, 2015; Published January 16,2015

Citation: Wang M, Wu B, Tucker JD, Lu P, Lu Q (2015) Tris[2-(Acryloyloxy) Ethyl]lsocyanurate Cross-Linked Polyethylenimine Enhanced Exon-Skipping of Antisense 2'-Omethyl Phosphorothioate Oligonucleotide in vitro and in vivo. J Nanomed Nanotechnol 6: 261. doi:10.4172/2157-7439.1000261

Copyright: $\odot 2015$ Wang M, et al. This is an open-access article distributed under the terms of the Creative Commons Attribution License, which permits unrestricted use, distribution, and reproduction in any medium, provided the original author and source are credited. 
Citation: Wang M, Wu B, Tucker JD, Lu P, Lu Q (2015) Tris[2-(Acryloyloxy)Ethyl]lsocyanurate Cross-Linked Polyethylenimine Enhanced ExonSkipping of Antisense 2'-Omethyl Phosphorothioate Oligonucleotide in vitro and in vivo. J Nanomed Nanotechnol 6: 261. doi:10.4172/21577439.1000261

uncharged antisense PMO in muscles by local administration $[28,29]$. PEAs (C series) composed of PEI 2.0k gave higher transfection efficiency compared with PEAs of PEI 0.8k (A series) or $1.2 \mathrm{k}$ (B series)constructed. These results have encouraged us to further investigate the potential of this polymer series for the exon-skipping of negatively charged antisense oligomer 2'-OMePS. The study validated that the PEA polymers apparently increased 2'-OMePS-induced exon-skipping efficiency over parent LPEIs and PEI 25k in vitro and in vivo. The PEAs composed of PEI 2.0k (C series) present the highest enhanced exonskipping as compared to B and A series. The most effective PEA C14, [TAEI-PEI 2.0k (1:4)] achieved up to 6 fold exon-skipping efficiency compared with 2'-OMePS only in $m d x$ mice via intramuscular injection without increasing muscle damage. The enhanced delivery efficiency and limited toxicity indicates the potential of these PEAs as nucleic acid delivery vehicles for gene/antisense therapy.

\section{Experimental Section}

\section{Materials}

The TAEI cross-linked LPEI polymers (PEAs) were prepared as described in our earlier publications $[28,29]$. Cell culture media Dulbecco'sModifiedEagle'sMedium(DMEM), fetalbovineserum(FBS), L-glutamine and HEPES buffer solution (1M), penicillin-streptomycin, and 3-(4,5-dimethylthiazol-2-yl)-5-(3-carboxymethoxyphenyl)2-(4-sulfophenyl)-2H-tetrazolium (MTS) were purchased from GIBCO, Invitrogen Corp (NY, USA). A single-stranded 20-mer deoxyoligonucleotide as a model antisense oligonucleotide $(\mathrm{AO})$ with the sequence $5^{\prime}$-GGCCAAACCTCGGCTTACCT-3' (phosphodiester) for physicochemical study of polymer/oligonucleotide polyplex; AOs modified by 2'-O-methylation and phosphorthioation 2'-OMePSE50 (5' - AACUUCCUCUUUAACAGAAAAGCAUAC - 3') targeting human dystrophin gene exon 50, 2'-OMePSE23 (5'-GGCCAAACCUCGGCUUACCU-3') targeting mouse dystrophin gene exon 23, and Cy3 labeled 2'-OMePS used for delivery study in vitro and in vivo were purchased from GenScript (NJ, USA). All other chemicals were purchased from Sigma-Aldrich (MO, USA), unless otherwise stated.

\section{Characterization of polymer/AO complexes}

Agarose gel electrophoresis: The polymer/AO complexes were prepared freshly before use by vortex of $\mathrm{AO}$ and polymer solutions at various weight ratios. The complexes were incubated at room temperature for 30 minutes in $25 \mu \mathrm{L}$ volume, and loaded on $1 \%$ agarose gel with ethidium bromide $(\mathrm{EB}, 0.1 \mu \mathrm{g} / \mathrm{mL})$. Gels were run with trisacetate (TAE $1 \mathrm{X}$ ) buffer at $100 \mathrm{~V}$ and analyzed on UV illuminator (Alphalmager HP, USA) to assess the location of the AOs.

Particle size and zeta potential determination by Dynamic Light Scattering (DLS): The hydrodynamic diameters of polymer/AO polyplexes in $0.9 \%$ sodium chloride were determined by DLS at a $90^{\circ} \mathrm{C}$ measurement angle with Zetaplu Zeta Potential Analyzer 90 Plus/BIMAS (Brookhaven Instrument Co. USA) equipped with a $15 \mathrm{mV}$ solidstate laser operated at a wavelength of $635 \mathrm{~nm}$. Briefly, $1 \mathrm{~mL}$ of polyplex solution containing $5 \mu \mathrm{g}$ of $\mathrm{AO}$ was prepared at various weight ratios (polymer $/ \mathrm{AO}=1 / 1$ up to $10 / 1$ ) and incubated 30 minutes at $4^{\circ} \mathrm{C}$, then the polyplex size was measured by photon correlation spectroscopy. For data analysis, the refractive index (1.33) and viscosity (0.89) of water at $25^{\circ} \mathrm{C}$ were used.

The zeta potential (surface charge) of polyplexes was determined at $25^{\circ} \mathrm{C}$ using the same apparatus equipped with $658 \mathrm{~nm}$ laser. The samples were diluted to 8 times with $\mathrm{diH}_{2} \mathrm{O}$ to ensure the measurement operated under low ionic strength, where the surface charge of particles can be accurately measured.

Morphology analysis by Transmission Electron Microscopy (TEM): The polymer/AO polyplex solution containing $1 \mu \mathrm{g}$ of $\mathrm{AO}$ was prepared at weight ratio of $5 / 1$ (polymer/AO) in $200 \mu \mathrm{L} 0.9 \% \mathrm{NaCl}$, pH 7.4 medium, and analyzed using TEM (Phillips CM-10, Philips Electronic North America Corp., Andover, MA, USA). The AO alone was used for comparison. The samples were prepared using negative staining with $1 \%$ phosphotungstic acid. Briefly, one drop of sample solution was placed on a formvar and carbon coated grid (Electron Microscopy Sciences, Hatfield, PA) for 1 hour, and the grid was blotted dry, followed by stained for 3 minutes. The grids were blotted dry again. Samples were analyzed at $60 \mathrm{kV}$. Digital images were captured with a digital camera system from 4 pi Analysis (Durham, NC, USA).

Polyplex stability: The relative stability of polyplexes was measured by measuring $\mathrm{AO}$ released from polyplexes in the presence of a competing polyanion-Heparin. Polyplex solutions were incubated 10 minutes in the presence of heparin (American Pharmaceutical Partners, Inc., Schaumburg, IL) over a range of concentrations at room temperature. All polyplexes were formed at weight ratio of polymer/ $\mathrm{AO}=5$, based on $1 \mu \mathrm{g}$ of AO. Samples were run on agarose gels $(1.0 \%$ in TAE buffer, $100 \mathrm{~V}, 20$ minutes), and the intensity of the "free AO" band was analyzed by densitometry on UV illuminator (Alphalmager $\mathrm{HP}$, USA) to assess the location of the AO. The percentage of AO dissociated from the polyplex was obtained by normalizing against the AO band of known amount on the same gel.

\section{Cell viability assay}

The cytotoxicity was evaluated in C2C12E50 cell line using the MTS [3-(4,5-dimethylthiazol-2-yl)-5-(3-carboxymethoxyphenyl)-2(4-sulfophenyl)-2H-tetrazolium]-based assay as described previously [29]. Briefly, the cells were seeded in a 96-well tissue culture plate at $1 \times 10^{4}$ cell per well in $200 \mu \mathrm{L} 10 \%$ FBS-DMEM medium. Cells achieving $70-80 \%$ confluence were exposed to polymer at different doses for 24 hours, followed by addition of $20 \mu \mathrm{L}$ of MTS solution. The viability was measured with Tecan 500 Plate reader (Tecan US, Inc, Morrisville, NC, USA). The relative cell viability was calculated by: $\left(\boldsymbol{A}_{\text {treated }}-\boldsymbol{A}_{\text {backround }}\right) \mathrm{x}$ $100 /\left(\boldsymbol{A}_{\text {control }}-\boldsymbol{A}_{\text {background }}\right)$. All viability assays were carried out in triplicate.

\section{Transfection in vitro}

The C2C12 E50 cell has a human dystrophin exon 50 sequence (hDysE50) placed inside the coding sequence of GFP gene under control of an actin promoter. The hDysE50 sequence is spliced out and the GFP coding sequence joined back in-frame through the functional effect of 2'-OMePS AO targeting E50.

C2C12 or $\mathrm{C} 2 \mathrm{C} 12$ E50 cells were cultured in 10\% FBS-DMEM medium and maintained at $37^{\circ} \mathrm{C}$ and $10 \% \mathrm{CO}_{2}$ in a humidified incubator. About $5 \times 10^{4}$ cells in $0.5 \mathrm{~mL} 10 \%$ FBS-DMEM medium were seeded in a 24 well plate and allowed to reach $70-80 \%$ confluence prior to transfection experiments. Cell culture medium was replaced prior to addition of polymer/2'-OMePS formulation at different weight ratios. PEI 25k was used as a control for delivery. Transfection efficiency indicated by GFP production, was visualized using an Olympus IX71 fluorescent microscope (Olympus America Inc., Milville, NY, USA) after 48 hours of incubation. Digital images were taken using the Olympus DP Controller and DP Manager software (Olympus America Inc., Milville, NY, USA). Transfection efficiency was also examined quantitatively on a FACS Calibur flow cytometer (BD, Franklin Lakes, NJ, USA). A minimum of $1 \times 10^{4}$ cells were counted and subsequently 
Citation: Wang M, Wu B, Tucker JD, Lu P, Lu Q (2015) Tris[2-(Acryloyloxy)Ethyl]lsocyanurate Cross-Linked Polyethylenimine Enhanced ExonSkipping of Antisense 2'-Omethyl Phosphorothioate Oligonucleotide in vitro and in vivo. J Nanomed Nanotechnol 6: 261. doi:10.4172/21577439.1000261

Page 3 of 9

analyzed with CellQuest Pro (BD, Franklin Lakes, NJ, USA) software package.

\section{Delivery in vivo}

This study was carried out in strict accordance with the recommendations in Guide for the Care and Use of Laboratory Animals of the National Institutes of Health. The protocols were approved by the Institutional Animal Care and Use Committee (IACUC), Carolinas Medical Center (Breeding protocol: 10-13-07A; Experimental protocol: 10-13-08A). All injection was performed under isoflurane anesthesia, and all efforts were made to minimize suffering.

Animals and intramuscular injections: Dystrophic $m d x$ mice aged 4-5 weeks were used for in vivo testing (5 mice each in test and control groups) unless otherwise stated.

The2'-OMePSE23(+02-18)(5'-GGCCAAACCUCGGCUUACCU-3') targeting the boundary sequences of exon and intron 23 of mouse dystrophin gene was used. For intramuscular (i.m.) injections, $5 \mu \mathrm{g}$ $2^{\prime}$-OMePSE23 with or without polymer was used in $40 \mu \mathrm{L}$ saline for each tibialis anterior (TA) muscle. Mice were sacrificed at two weeks after treatment, and collected muscles were snap-frozen in liquid nitrogencooled isopentane and stored at $-80^{\circ} \mathrm{C}$.

Reverse transcription polymerase chain reaction (RTPCR): Total RNA was extracted from TRIzol after dissection, and $100 \mathrm{ng}$ of RNA template was used for a $50 \mu \mathrm{L}$ RT-PCR reaction with the Stratascript One-Tube RT-PCR System (Stratagene, Santa Clara, CA). The primer sequences for the RT-PCR were Ex20Fo 5'-CAGAATTCTGCCAATTGCTGAG-3' and Ex26Ro 5'TTCTTCAGCTTGTGTCATCC-3' for amplification of mRNA from exons 20 to 26 . A total of 40 cycles were carried out for the RT-PCR. Bands with the expected size for the transcript with exon 23 deleted were extracted and sequenced. The intensity of the bands of PCRamplified products obtained from the treated muscles was measured with the National Institutes of Health (NIH) ImageJ software 1.42, and the percentage of exon-skipping was calculated with the intensity of the 2 bands representing both unskipped and skipped exons as $100 \%$.

Antibodies, immunohistochemistry, and western blots: Sections of $6 \mu \mathrm{m}$ were cut from the muscles and stained with a rabbit polyclonal antibody P7 for the dystrophin protein and detected by goat antirabbit Igs Alexa 594 (Invitrogen, NY, USA). The maximum number of dystrophin-positive fibers in one section was counted using the Olympus IX51 fluorescent microscope (Olympus America Inc., Milville, NY, USA). Digital images were taken with the Olympus DP Controller and DP Manager software (Olympus America Inc., Milville, NY, USA) and the muscle fibers were defined as dystrophin-positive when more than two-thirds of the membrane of a single fiber showed continuous staining.

Protein extraction and Western blot were performed as described previously $[7,11,26,29]$. Briefly, the membrane was probed with NCLDYS1 monoclonal antibody against dystrophin rod domain (Vector Laboratories, Burlingame, CA, USA) followed by HRP-conjugated goat anti-mouse IgG (Santa Cruz Biotechnology, Santa Cruz, CA, USA) and the ECL ${ }^{\mathrm{TM}}$ Western Blotting Analysis System (Perkin-Elmer, Waltham, MA, USA). The intensity of the bands with appropriate size was measured and compared with that from normal muscles of C57BL mice using NIH ImageJ software. Loading control of $\alpha$-Actin was detected by rabbit anti-actin antibody (Sigma, St. Louis, MO, USA).

\section{Statistical analysis}

All data are reported as mean \pm SD. Statistical differences between treatment and control groups were evaluated by Student's $t$-test. Statistical significance was accepted when $p \leq 0.05$.

\section{Results and Discussion}

\section{Synthesis and characterization of PEA polymers [28,29]}

The PEA polymers were designed and prepared from Polyethylenimine(s) (PEI, Mw: $0.8 \mathrm{k} / 1.2 \mathrm{k} / 2.0 \mathrm{kDa}$ ) and Tris[2(acryloyloxy)ethyl]isocyanurate (TAEI) by Michael addition reaction [30]. The products were dialyzed using Spectra/Por membrane (molecular mass cutoff $2 \mathrm{k} \mathrm{Da}$ ) against 20\% methanol and distilled water, then lyophilized. The composition and molecular weight were characterized with ${ }^{1} \mathrm{H}$ NMR JEOL500 spectrometer and Capillary viscosity, respectively. The microstructures and compositions of these cationic amphiphilic polymers are illustrated in Scheme 1 and Table 1, respectively.

\section{Characterization of polymer/AO polyplex}

To determine whether the PEA polymers bind AO, we examined the mixture of the two components at various ratios by agarose gel electrophoresis. Electrostatic interaction between the negatively

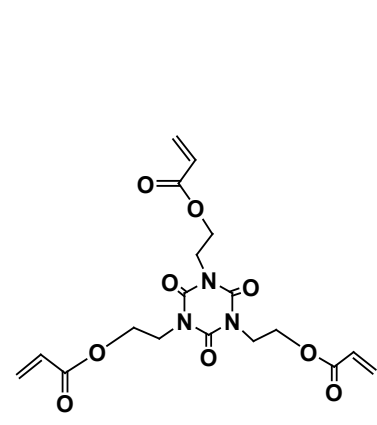

Tris(2-(acryloyloxy)ethyl] isocyanurate $M w=423.37$

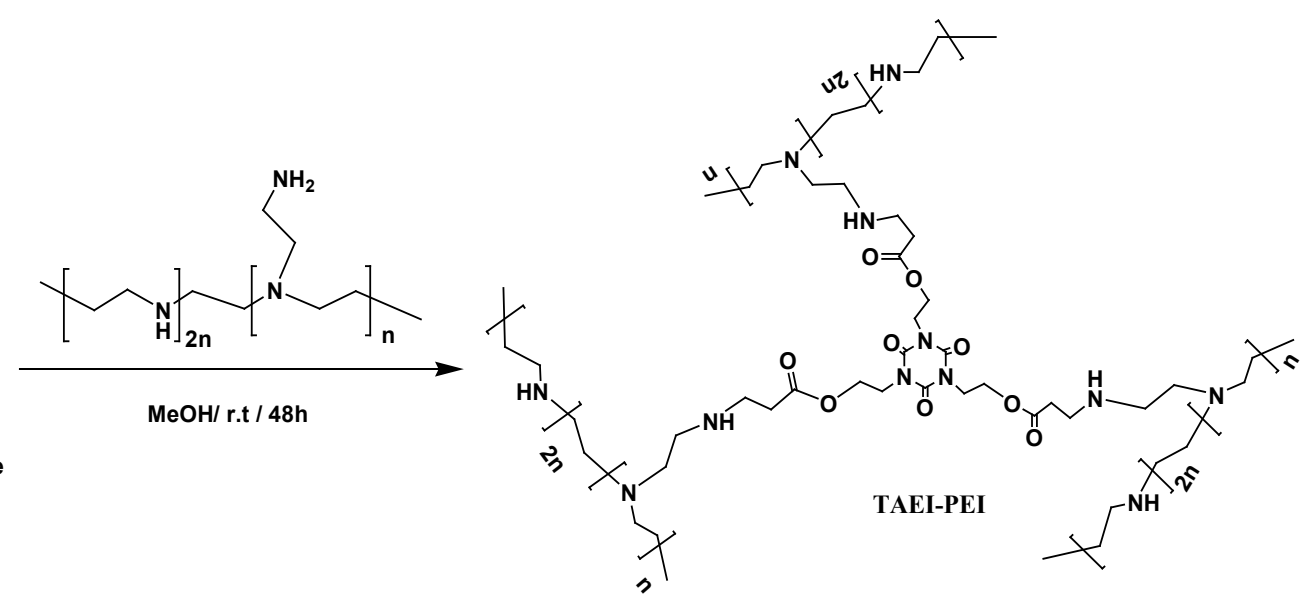

Scheme 1: Synthesis of PEAs based on TAEI cross-linked LPEI. 
Citation: Wang M, Wu B, Tucker JD, Lu P, Lu Q (2015) Tris[2-(Acryloyloxy)Ethyl]lsocyanurate Cross-Linked Polyethylenimine Enhanced ExonSkipping of Antisense 2'-Omethyl Phosphorothioate Oligonucleotide in vitro and in vivo. J Nanomed Nanotechnol 6: 261. doi:10.4172/21577439.1000261

Page 4 of 9

\begin{tabular}{|c|c|c|c|c|c|c|}
\hline Series & Code & Mole ration of TAEI/PEI & Mw PEI(Da) & $M v^{a}$ & PEI mol (\%) & Yield of copolymer (\%) \\
\hline \multirow{3}{*}{ A } & A11 & $1: 1$ & 800 & 4980 & 63.2 & 28.1 \\
\hline & A12 & $1: 2$ & 800 & 4570 & 72.5 & 32.4 \\
\hline & A14 & $1: 4$ & 800 & 4390 & 53.4 & 40.5 \\
\hline \multirow{3}{*}{ B } & B11 & $1: 1$ & 1200 & 7160 & 51.7 & 33.6 \\
\hline & B12 & $1: 2$ & 1200 & 5940 & 70.3 & 37.8 \\
\hline & B14 & $1: 4$ & 1200 & 5580 & 63.5 & 43.3 \\
\hline \multirow{3}{*}{ C } & $\mathrm{C} 11$ & $1: 1$ & 2000 & 9870 & 54.8 & 38.5 \\
\hline & $\mathrm{C} 12$ & $1: 2$ & 2000 & 8430 & 65.6 & 41.3 \\
\hline & $\mathrm{C} 14$ & $1: 4$ & 2000 & 7650 & 71.4 & 53.2 \\
\hline
\end{tabular}

$\mathrm{PEI}$, polyethylenimine; TAEI, tris[2-(acryloyloxy)ethyl]isocyanurate.

aDetermined by viscosity measurements in $0.9 \% \mathrm{NaCl}$ solution at $25^{\circ} \mathrm{C}$

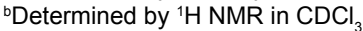

Table 1: Characteristics of synthesized PEAs.

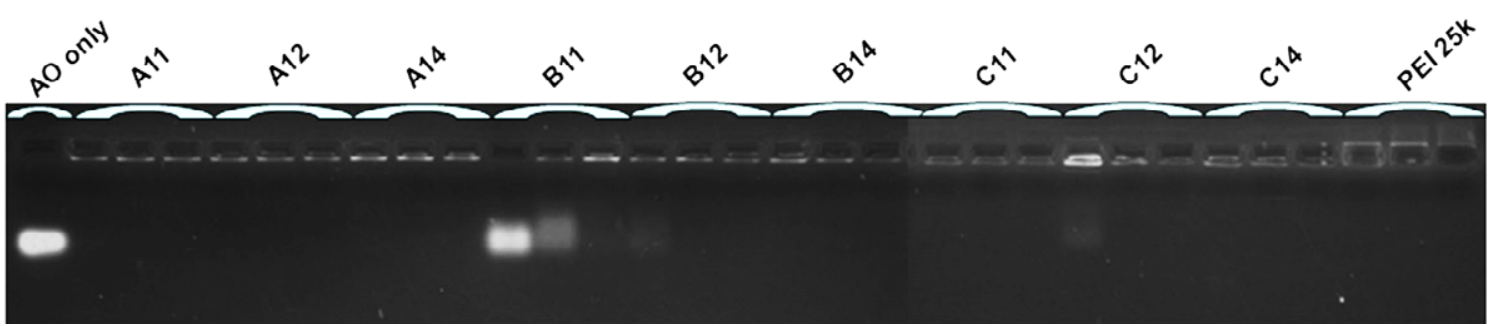

Figure 1: Electrophoretic mobility of polymer/AO polyplexes at three weight ratios, $0.5: 1,1: 1$ and $2: 1$ (from left to right for each polymer). $1 \mu \mathrm{g} A O$ in total of $25 \mu \mathrm{L}$ medium was used. The first left lane is loaded with $1 \mu \mathrm{g} \mathrm{AO}$ only control for comparison.

charged $\mathrm{AO}$ and the positively charged polymers leads to subsequent condensation and polyplex formation, along with retarded migration within the gel. As shown in Figure 1, nearly all PEA polymers used with $\mathrm{AO}$ at ratios 1:1 or higher (except for B11) showed strong binding ability similar to PEI $25 \mathrm{k}$ performance in the test dosages resulting in condensed and/or charge-neutralized polymer/AO complexes, indicated by the shifting of the EB stained bands towards markedly higher mass positions, with most remaining within the loading wells in the agarose gel. These results are consistent with the expectation that negatively charged AO are progressively neutralized by the positive charges of PEA as the ratio of PEA/AO increased. The comparably weak binding of the polymer B11 to AO is likely due to the higher molecular weight $(\mathrm{Mw})$, but lower PEI content compared to other PEAs in the corresponding series (such as B12, B14). These results also demonstrated that binding affinity of the PEAs to short $\mathrm{AO}$ is stronger than to pDNA under the same conditions as we reported previously [28]. The results further indicate that both the polymer size and positive charge components are critical factors for the condensation of negatively charged $\mathrm{AO}$ or $\mathrm{pDNA}$.

The particle size of polymer/AO complex is an important factor for effective delivery. We examined herein the complex by DLS, and found that all of the tested polymers condensed oligonucleotide into nano-sized particles at the polymer/AO $(\mathrm{w} / \mathrm{w})$ ratio of $2: 1$ and above. As expected, the average surface charges of polyplexes at the tested ratio ranged from -17.2 to $8.5 \mathrm{mV}$ (the polymer/AO weight ratio from 1 to10), with an average particle size below $260 \mathrm{~nm}$ at the tested ratio range. Notably the particle sizes were between 60 $150 \mathrm{~nm}$ for all the complexes at the ratio of polymer $/ \mathrm{AO}=5: 1$. The mean particle sizes of the C14/AO complex was $67 \mathrm{~nm}$ with relatively narrow distribution (Figure 2). To obtain further insight into polyplex characteristics, the morphology was studied by TEM using the same polymer/AO formulation at weight ratio of 5:1. The polyplex particles were uniformly distributed with sizes below $100 \mathrm{~nm}$. The polyplexes of $\mathrm{C} 12$ or $\mathrm{C} 14$ with $\mathrm{AO}$ were spherical with size distribution around 30-60 nm. The particles size of C14/AO complex were slightly smaller than that of C12/AO complex, possibly because of the relatively lower $\mathrm{Mw}$ and higher PEI contents within polymer C14 compared with C12. The oligonucleotides alone formed heterogeneous particles with size ranging from less than $10 \mathrm{~nm}$ to over $100 \mathrm{~nm}$. These results support the view that density and the number of cationic units within polymers play a key role in the formation of polymer/AO complex [31].

\section{Polyplex stability in the presence of polyanion}

We chose heparin as a model compound to evaluate polymer/ AO polyplex stability in the presence of polyanion. Heparin, a highly sulfated glycosaminoglycan (GAG), is a negatively charged linear polysaccharide. GAGs are major components within the extracellular matrix of many tissues, but also found within, and on cell surfaces [32]. To evaluate polyplex stability, polyplexes were incubated with varying amounts of heparin prior to electrophoresis in agarose gels (Figure 3). All polyplexes contained the same amount of $\mathrm{AO}(1 \mu \mathrm{g})$ and were prepared at the same ratio of polymer/AO $(\mathrm{w} / \mathrm{w}=5)$. $\mathrm{AO}$ was not released from all the polyplexes and remained visible within the sample wells when no heparin was added, indicating that the polyplexes at the tested dose ratio were sufficiently stable under electrophoretic condition. In the presence of $25 \mu \mathrm{g} / \mathrm{mL}$ sodium heparin, EB signals remained in the loading well in all samples of polymer/AO plyplex except for B11/AO polyplex which showed a weak band of the same size as $\mathrm{AO}$ alone. The dissociation of $\mathrm{AO}$ from the polyplex with $\mathrm{B} 11$ and $\mathrm{C} 12$ were about $30 \%$, and $40 \%$ respectively, as increasing the concentration of sodium heparin to $50 \mu \mathrm{g} / \mathrm{mL}$. Almost $100 \%$ AO was dissociated from the $\mathrm{B} 11, \mathrm{C} 12 / \mathrm{AO}$ polyplexes when the heparin concentration goes to $100 \mu \mathrm{g} / \mathrm{mL}$; while other polymer composed polyplexes still remained intact comparable to PEI-based polyplex in such high concentration of sodium heparin. These results were coincident with the binding potential of polymer-AO, indicating that 
Citation: Wang M, Wu B, Tucker JD, Lu P, Lu Q (2015) Tris[2-(Acryloyloxy)Ethyl]lsocyanurate Cross-Linked Polyethylenimine Enhanced ExonSkipping of Antisense 2'-Omethyl Phosphorothioate Oligonucleotide in vitro and in vivo. J Nanomed Nanotechnol 6: 261. doi:10.4172/21577439.1000261

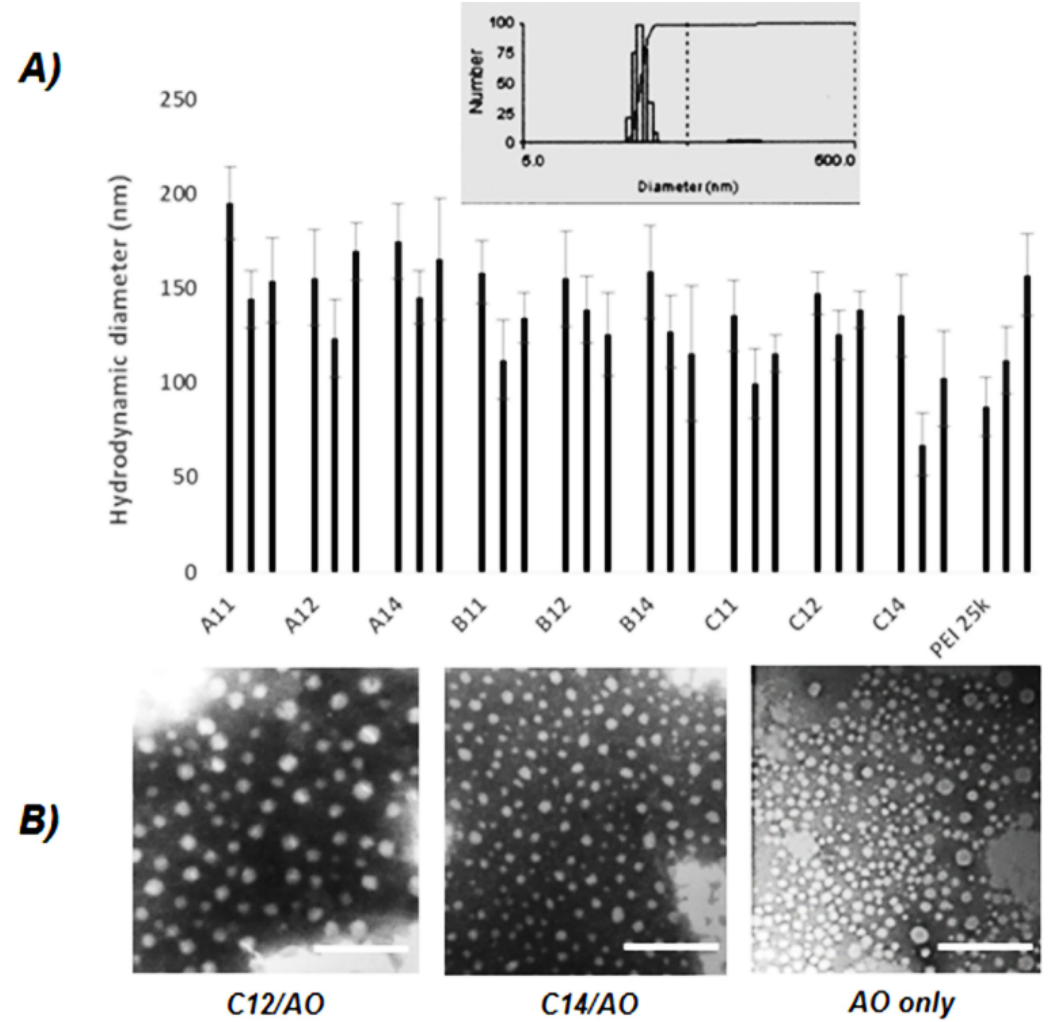

Figure 2: Biophysical properties of polymer/AO polyplex. A) Particle size and distribution of polymer/AO at three weight ratios (2/5/10:1); B) Negative staining TEM images of polymer/AO (5/1) complexes (scale bar $200 \mathrm{~nm}$ ).

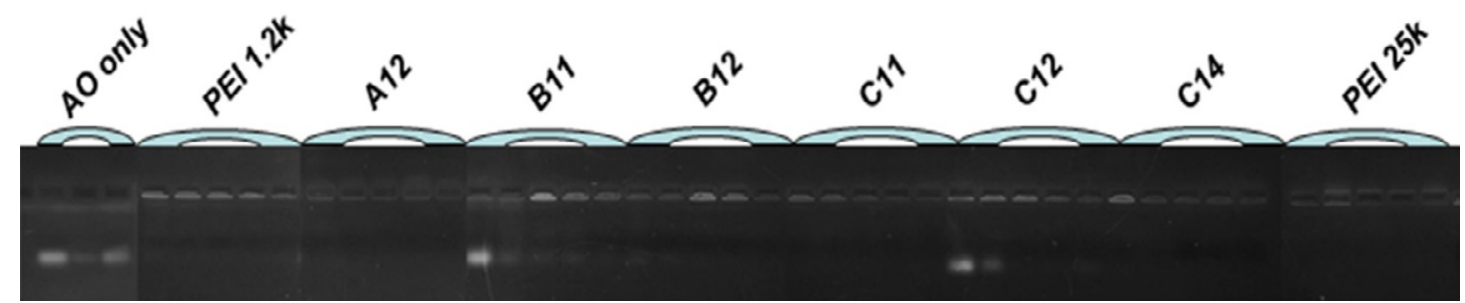

Figure 3: Stability of polyplexes in the presence of heparin. Polyplexes containing PEA complexed with AO were incubated with varying amounts of heparin prior to separation on agarose gels. All polyplexes contained $1 \mu \mathrm{g}$ of $\mathrm{AO}$, and were prepared at the same weight ratio of polymer/AO $=5$. The full range of heparin concentrations examined $(100,50,25,10,0 \mu \mathrm{g} / \mathrm{mL}$ from left to right for each sample).

the stronger binding affinity of the polymer/AO complex resulted in higher resistance to the polyanion. By comparing PEI $1.2 \mathrm{~K}$ versus $\mathrm{B}$ series, the results suggest the presence of TAEI in the PEAs weakens the binding of LPEI to oligonucleotide. The concentration dependent release of oligonucleotide from polymer/AO complex in the presence of highly negative charged heparin, the abundance of which varies considerably among tissues, may be useful to design polymer-based vectors for controlled release of delivery cargo.

Regardless of the mechanism, the heightened stability of PEA/AO polyplexes has important implications for their effectiveness as $\mathrm{AO}$ delivery carriers. The PEA/AO polyplexes were more stable than those of PEA/pDNA under similar test condition, which is in accordance with the binding affinity results [28]. These findings underscore the need to verify the stability of polyplexes and delivery cargo structures used in transfections, both of which are key determinants of polyplex properties including efficacy as delivery vectors.

\section{Evaluation of $2^{\prime}$-OMePS delivery in $\mathrm{C} 2 \mathrm{C} 12$ myoblasts expressing GFP/hDysE50}

The cytotoxicity of PEAs in C2C12E50 cells were evaluated and reported previously [29]. All PEAs except C11 [TAEI-PEI 2.0k (1:1)], at a dose of $20 \mu \mathrm{g} / \mathrm{mL}$ showed cell viability over $80 \%$, higher than PEI $25 \mathrm{k}$ at $4 \mu \mathrm{g} / \mathrm{mL}$. C11 had the highest toxicity among all PEAs, but still owned over $67 \%$ living cell at high concentration of $20 \mu \mathrm{g} / \mathrm{mL}$. The relatively higher toxicity of $\mathrm{C} 11$ is likely due to its higher molecular weight, and the relatively high lipophilicity due to its lower PEI composition when compared with other PEAs. Generally, the more lipophilic the polymer is, the higher the toxic is both in vitro and in vivo [26-29]. Based on the cytotoxicity data, we examined the effect of PEA polymers on exon-skipping at four different doses $(4,10,20$, and $40 \mu \mathrm{g} / \mathrm{mL})$ with 
Citation: Wang M, Wu B, Tucker JD, Lu P, Lu Q (2015) Tris[2-(Acryloyloxy)Ethyl]lsocyanurate Cross-Linked Polyethylenimine Enhanced ExonSkipping of Antisense 2'-Omethyl Phosphorothioate Oligonucleotide in vitro and in vivo. J Nanomed Nanotechnol 6: 261. doi:10.4172/21577439.1000261

Page 6 of 9

$2^{\prime}$-OMePS $(4 \mu \mathrm{g} / \mathrm{mL})$ in the C2C12E50 cell line. A 2'-OMePS sequence, 2'-OMePSE50 (5' - AACUUCCUCUUUAACAGAAAAGCAUAC $\left.3^{\prime}\right)$ targeting the inserted hDysE50 was used. The expression of GFP in the cells is interrupted by the insertion of the hDysE50 within the GFP coding sequence. The restoration of GFP expression depends on the targeted removal of the exon 50 by $2^{\prime}$-OMePSE50. The cells were treated with the $2^{\prime}$-OMePSE50 at a fixed amount $(2 \mu \mathrm{g})$ formulated with each polymer in $0.5 \mathrm{~mL} 10 \%$ FBS-DMEM medium. The transfection efficiency (TE) of PEAs-mediated 2'-OMePSE50 was determined by fluorescence microscopy analysis two days after transfection. The results showed almost all PEA polymers at $10 \mu \mathrm{g} / \mathrm{mL}$ produced enhanced GFP expression as compared to the 2'-OMePS alone. The highest levels of GFP expression were achieved at the dose of $20 \mu \mathrm{g} / \mathrm{mL}$ with most PEAs (data not shown here), whereas less cells were gauged as GFP positive when treated with $2^{\prime}$-OMePS itself. The enhanced exon-skipping was consistent with the dramatically increased signal intensity of the $\mathrm{Cy} 3$ labeled 2'-OMePS (Cy3-Oligo) within C2C12 cells in the presence of PEAs (polymer $10 \mu \mathrm{g}$, and Cy3-Oligo $1 \mu \mathrm{g}$ in $0.5 \mathrm{~mL} \mathrm{10 \%} \mathrm{FBS-DMEM}$ medium) compared with PEI 25k-mediated ( $2 \mu \mathrm{g}$ used due to high toxicity at higher dose) or Cy3-Oligo alone (Figure 4A). The delivery efficiency and cell toxicity of $2^{\prime}$-OMePSE50 $(2 \mu \mathrm{g})$ formulated with PEAs $(10 \mu \mathrm{g})$ in $0.5 \mathrm{~mL} 10 \%$ FBS-DMEM medium for C2C12E50 cell line was measured qualitatively and quantitatively by fluorescence microscopy and flow cytometry, respectively (Figures 4B-4D). The efficiency of enhanced exon-skipping with the polymers of $\mathrm{C}$ series was clearly higher than that of the A or B series and PEI 25k, especially with the $\mathrm{C} 12$ and $\mathrm{C} 14$ achieving over $60 \%$ with lower cytotoxicity. In contrast, the exon-skipping with PEI 25k/2'-OMePS and 2 '-OMePS alone gave around $10 \%,<1.5 \%$, respectively. LPEI alone-formulated $2^{\prime}$-OMePS produced less GFP expression close to $2^{\prime}$-OMePS only (data not shown here). Cytotoxicity of the PEA/2'-OMePS complexes was less than that of PEI $25 \mathrm{k} / 2^{\prime}$-OMePS (Figures $4 \mathrm{C}-4 \mathrm{E}$ ). These results

A)

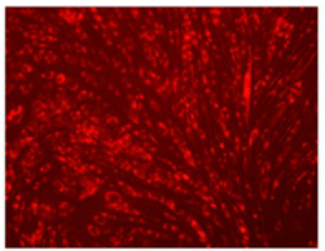

$\mathrm{C} 14,10 \mu \mathrm{g}, 48 \mathrm{~h}$

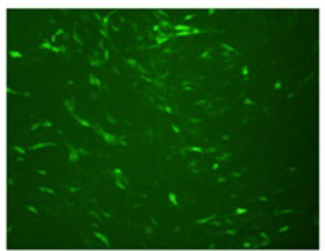

$\mathrm{C} 14,10 \mu \mathrm{g}, 48 \mathrm{~h}$

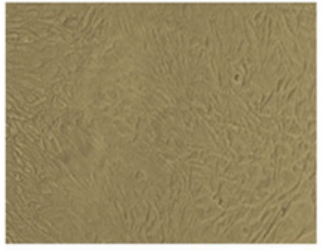

$\mathrm{C} 14,10 \mu \mathrm{g}, 48 \mathrm{~h}$

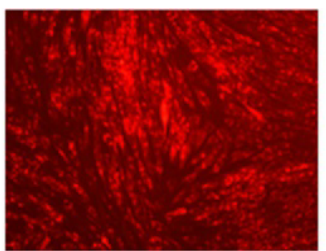

$\mathrm{C} 12,10 \mu \mathrm{g}, 48 \mathrm{~h}$

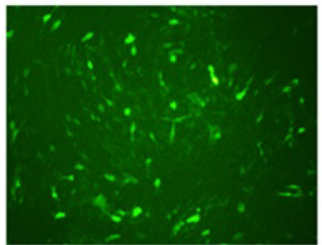

$\mathrm{C} 12,10 \mu \mathrm{g}, 48 \mathrm{~h}$

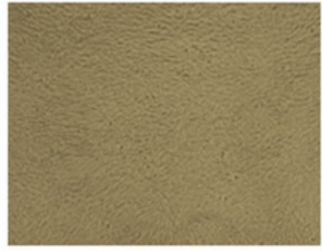

Cell only, $48 \mathrm{~h}$

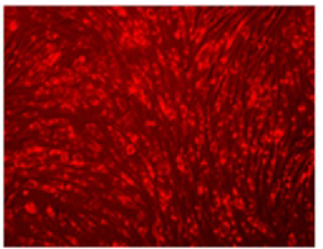

B12, $10 \mu \mathrm{g}, 48 \mathrm{~h}$

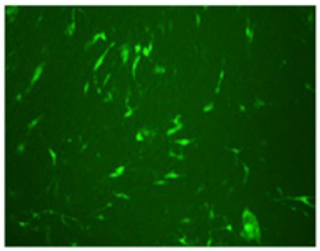

$\mathrm{B} 12,10 \mu \mathrm{g}, 48 \mathrm{~h}$

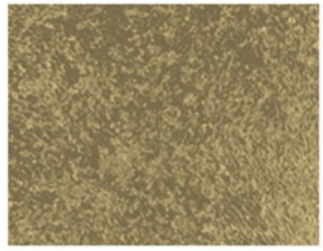

B12, $10 \mu \mathrm{g}, 48 \mathrm{~h}$

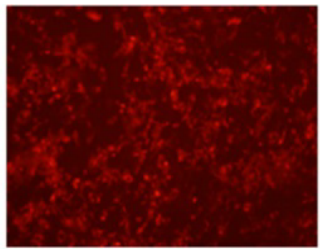

PEI 25k $2 \mu \mathrm{g}, 48 \mathrm{~h}$

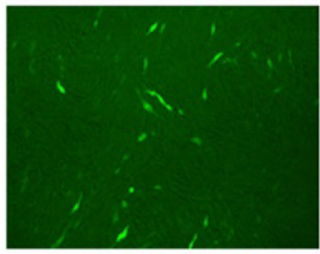

PEI $25 \mathrm{k}, 2 \mu \mathrm{g}, 48 \mathrm{~h}$

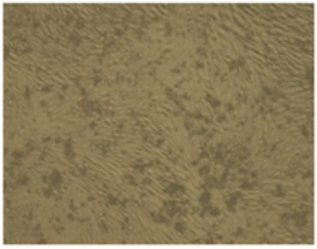

PEI 25k, $2 \mu \mathrm{g}, 48 \mathrm{~h}$

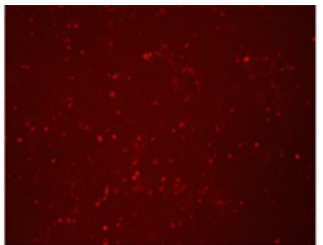

Cy3-oligo, 48h

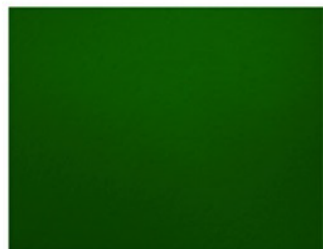

2'-OMePS, $2 \mu \mathrm{g}, 48 \mathrm{~h}$

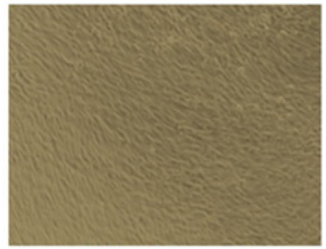

2'-OMePS, $2 \mu \mathrm{g}, 48 \mathrm{~h}$

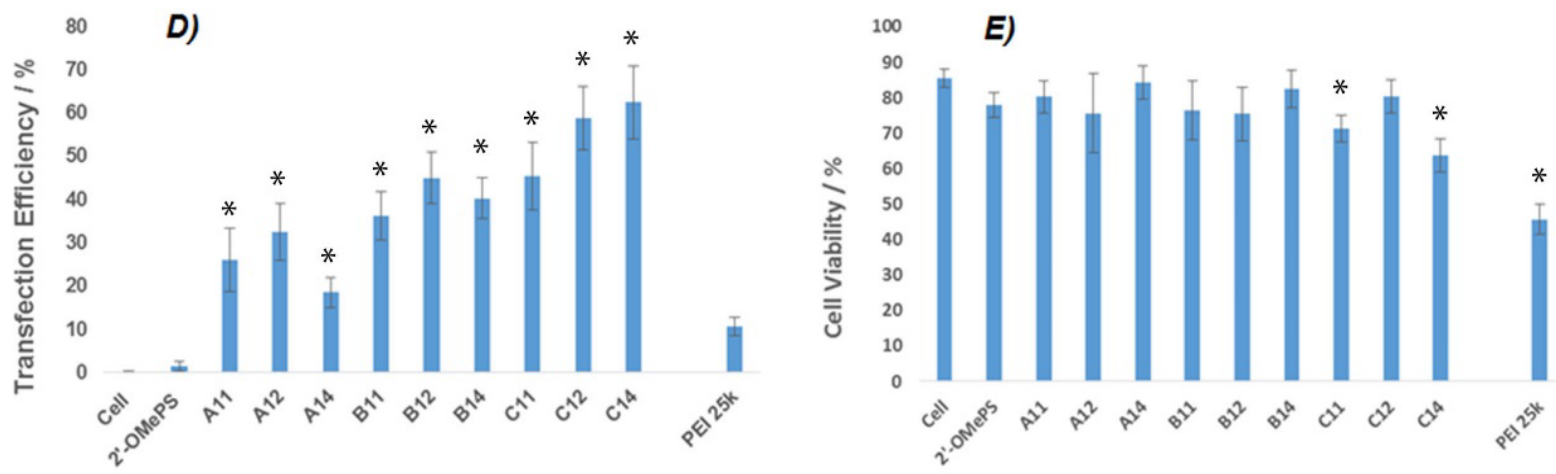

Figure 4: A) Cy3 labeled 2'-OMePS (Cy3-Oligo) with and without polymer formulation in C2C12 cell line. B) Representative fluorescence images of 2'-OMePSE50 -induced exon-skipping in C2C12E50 cell line. C) Cell morphology under bright field. 2'-OMePSE50 (2 $\mu \mathrm{g})$ formulated with PEA polymers (10 $\mu \mathrm{g})$, PEI $25 \mathrm{k}(2 \mu \mathrm{g})$ in $0.5 \mathrm{~mL} 10 \%$ FBS-DMEM. The images were taken $48 \mathrm{~h}$ after treatment. Original magnification, $\mathrm{x} 100$. D) Statistical date of TE of $2^{\prime}-\mathrm{OMePSE} 50$ (2 $\left.\mu \mathrm{g}\right)$ formulated with polymers $\left(\mathrm{n}=3\right.$, two-tailed t-test, ${ }^{*} p \leq 0.05$ compared with $2 \mu \mathrm{g} 2^{\prime}$-OMePS only). E) Cell viability with $2^{\prime}-\mathrm{OMePSE} 50$. The results are presented as the mean \pm SD in triplicate ( $\mathrm{n}=3$, two-tailed t-test, ${ }^{*} p \leq 0.05$ compared with untreated cell as control). 
Citation: Wang M, Wu B, Tucker JD, Lu P, Lu Q (2015) Tris[2-(Acryloyloxy)Ethyl]lsocyanurate Cross-Linked Polyethylenimine Enhanced ExonSkipping of Antisense 2'-Omethyl Phosphorothioate Oligonucleotide in vitro and in vivo. J Nanomed Nanotechnol 6: 261. doi:10.4172/21577439.1000261

Page 7 of 9

further confirm the low cytotoxicity of PEAs, which has been observed in different cell line for different delivery cargos $[28,29]$.

\section{Delivery of $2^{\prime}$-OMePS with PEAs in $m d x$ mice}

The effect of the PEA polymers for 2'-OMePS delivery in vivo were evaluated by i.m. injection with the $2^{\prime}$-OMePS E23 targeting mouse dystrophin exon 23 (mDysE23) to each TA muscles of the $m d x$ mice aged 4-5 weeks. The $m d x$ mouse contains a nonsense mutation in the exon 23, preventing the muscle from producing functional dystrophin protein. Targeted removal of the mutated exon 23 is able to restore the reading-frame of dystrophin transcripts, and thereby the expression of functional dystrophin protein. All PEA polymers were examined at the dose of $5 \mu \mathrm{g}$ complexed with $5 \mu \mathrm{g}$ of $2^{\prime}$-OMePSE23 in $40 \mu \mathrm{L}$ saline, with the treated TA muscles being collected 2 weeks after treatment.
Immunohistochemistry showed that 2'-OMePSE23 alone induced a maximum of $29 \pm 5$ dystrophin positive fibers in one cross-section of the treated muscle. The induced numbers of dystrophin-positive fibers increased up to 2-6 folds in the muscles treated with PEAs-medicated 2'-OMePSE23. In particular, dystrophin positive fibers were counted to $75 \pm 9,102 \pm 15,125 \pm 21$ and $170 \pm 16$ in the muscles treated with B12, C11, C12 and C14 formulated 2'-OMePSE23, respectively. This is consistent with the higher efficiency of PEI 2.0k-based PEAs (C11, $\mathrm{C} 12, \mathrm{C} 14)$ in exon-skipping observed in cell culture. However, PEI 25k-formulated 2 -OMePSE23 produced only $45 \pm 8$ positive fibers. The enhance numbers of dystrophin positive fibers with B11-mediated 2'-OMePSE23 was lower as compared to others, probably due to lower PEI content, thus poor stability of B11/2'-OMePS complex at this dose ratio (Figure 5). The exon-skipping performance and dystrophin expression were also examined by RT-PCR and Western-blot. The

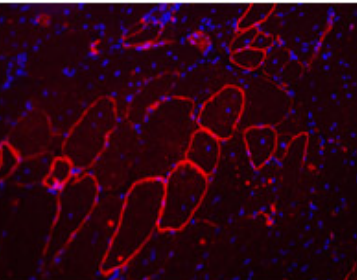

A12

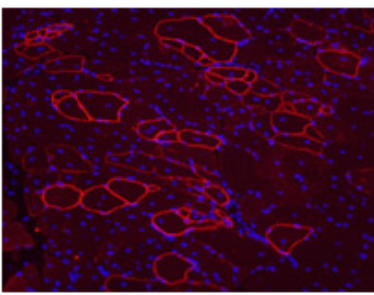

C11

b)

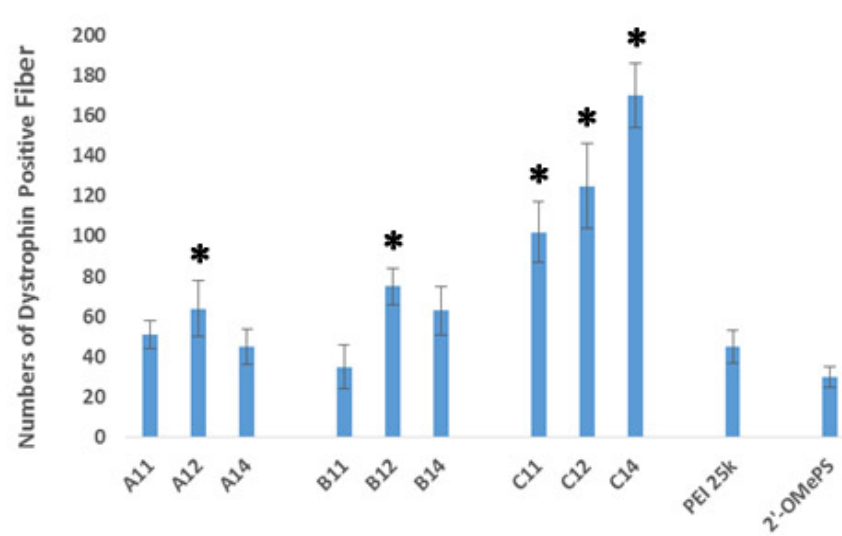

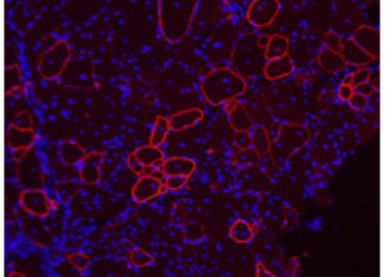

B12

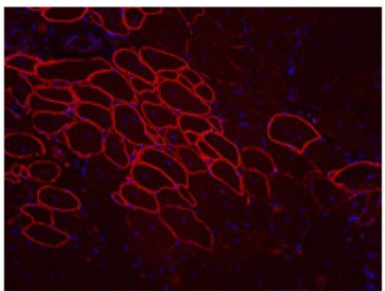

C14

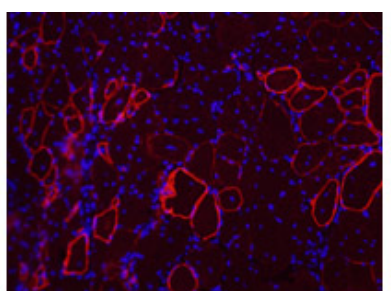

B14

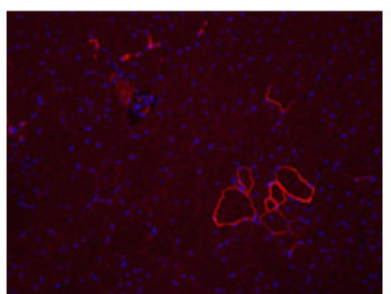

2-'OMePS only

c)
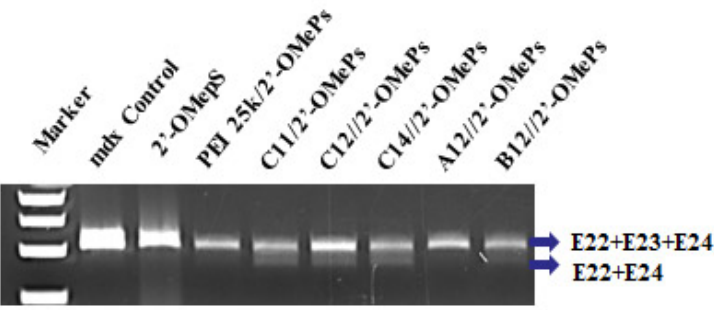

d)

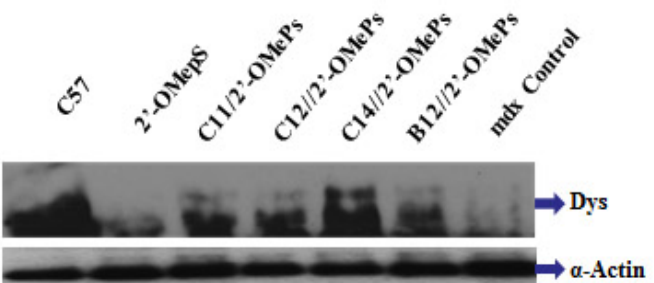

Figure 5: Dystrophin exon-skipping and protein expression following i.m. administration of 2'-OMePSE23 with and without polymers in TA muscle of $m d x$ mice (aged 4-5 weeks) 2 weeks after treatment. Muscles were treated by local injection with 2'-OMePSE23 (5 $\mu \mathrm{g})$ and polymer ( $5 \mu \mathrm{g})$ in $40 \mu \mathrm{L}$ saline. 2'-OMePSE23 alone and PEI $25 \mathrm{k}$ were used as controls: a) Restoration of dystrophin in TA muscles was detected by immunohistochemistry with rabbit polyclonal antibody P7 against dystrophin. Blue nuclear staining with DAPI (4,6-diamidino-2-phenylindole). Original magnification, $x$ 40. b) The numbers of dystrophin-positive fibers. The maximum numbers of dystrophin-positive fibers were counted in a single cross-section ( $n=5$, two-tailed t-test, * $p \leq 0.05$ compared with $5 \mu \mathrm{g} 2$ '-OMePSE23). $c$ ) Detection of exon 23 skipping by RT-PCR. Total RNA of $100 \mathrm{ng}$ from each sample was used for amplification of dystrophin mRNA from exon 20 to exon 26 . The upper bands (indicated by E22+E23+E24) correspond to the normal mRNA and the lower bands (indicated by E22+E24) correspond to the mRNA with exon E23 skipped. d) Western blotting demonstrate the expression of dystrophin protein from treated $m d x$ mice in comparison with C57BL/6 and untreated $m d x$ mice. Dys, dystrophin detected with monoclonal antibody Dys 1. a-Actin was used as the loading control. 
Citation: Wang M, Wu B, Tucker JD, Lu P, Lu Q (2015) Tris[2-(Acryloyloxy)Ethyl]lsocyanurate Cross-Linked Polyethylenimine Enhanced ExonSkipping of Antisense 2'-Omethyl Phosphorothioate Oligonucleotide in vitro and in vivo. J Nanomed Nanotechnol 6: 261. doi:10.4172/21577439.1000261

Page 8 of 9

levels of exon-skipping were about $28.3 \%, 18.7 \%, 31.6 \%, 3.9 \%, 22.3 \%$, $1.2 \%$ and $4.9 \%$ for $\mathrm{C} 11, \mathrm{C} 12, \mathrm{C} 14, \mathrm{~A} 12, \mathrm{~B} 12$, PEI 25k and 2'-OMePS only, respectively. Dystrophin protein expression levels were found to be around $36.9 \%, 34.6 \%, 82.3 \%, 41.8 \%$, and $2.7 \%$ of normal levels for $\mathrm{C} 11, \mathrm{C} 12, \mathrm{C} 14, \mathrm{~B} 12$, and $2^{\prime}$-OMePS only, respectively against $100 \%$ for $\mathrm{C} 57$. The quantitative data in vivo demonstrated that the $\mathrm{C}$ series (PEI-2k constructed PEAs) resulted in better delivery performance compared with $\mathrm{A}$ or $\mathrm{B}$ series, with $\mathrm{C} 14$ being the best for delivering anionic 2 '-OMePS. It is possible that cationic carriers must contain enough charge to neutralize the negative $\mathrm{AO}$, while also to provide sufficient residual charge for interaction with the cell membrane. This reflects the difficulty in finding optimal delivery conditions that are relevant to a vector's molecular composition, size, charges, and delivery cargo's chemical structure, as well as the optimal weight or N/P ratios. Hematoxylin and Eosin (H\&E) staining revealed no signs of increased inflammation, tissue remodeling, or fiber necrosis compared with saline-treated $m d x$ muscles as control, further confirming that these polymers were minimally cytotoxic as demonstrated in vitro and in vivo $m d x$ mice for $\mathrm{pDNA}$ and PMO delivery $[28,29]$.

\section{Conclusions}

In this study, the branched poly(ester amine)s (PEAs) based on Tris[2-(acryloyloxy)ethyl]isocyanurate (TAEI) cross-linked low molecular weight polyethylenimine (LPEI, Mw: $0.8 \mathrm{k} / 1.2 \mathrm{k} / 2.0 \mathrm{k}$ ) have been evaluated for the first time for the ability to deliver negativelycharged antisense $2^{\prime}$-OMePS in vitro and in dystrophic $m d x$ mice. All the PEAs were found to enhance the delivery of $2^{\prime}$-OMePS with the highest efficiency achieved by PEA-C series (constructed with TAEI and PEI 2.0k), and the maximum targeted exon-skipping by C14 [TAEIPEI 2.0k (1:4)]. Results from the current and our previous studies together show that PEA-C series produce significant enhancement in delivery efficiency for three nucleic acid cargos: pDNA [28], PMO [29] and 2'-OMePS. C12 [TAEI-PEI 2.0k (1:2)] produced highest delivery efficiency for pDNA and charge-neutral oligonucleotide PMO; while the $\mathrm{C} 14$ gave the highest delivery efficiency for anionic antisense oligonucleotide 2'-OMePS. 2'-OMePS and pDNA are similar in structure and charge, but are varied in their molecular size and unit linkage. The anionic 2 -OMePS oligonucleotides and more cationic polymer C14 (PEI $\%=71.4 \%, \mathrm{Mw}=7,650)$ containing higher PEI content compared with C12 (PEI $\%=65.6 \%, \mathrm{Mw}=8,430)$ are likely to build more stable complex, particularly when the complex is presented within a biological system and interacts with serum and many other cellular components. The relatively hydrophobic nature of 2'-OMePS would mean that the force between the polymer and the oligonucleotides are not only from the Coulomb interaction, but also from the hydrophobic interaction and hydrogen bonding. The drivingforce for the complex formation of the polymer with uncharged PMO would mainly come from the hydrophobic interaction and hydrogen bonding between them. This might well explain the higher transfection efficiency with $\mathrm{C} 12$ than with $\mathrm{C} 14$ for PMO delivery. The delivery performances of PEAs for different cargos therefore depend on the electrostatic interaction, hydrogen bonding and hydrophobic interaction related to the PEI size, PEI content, the composition and HLB of overall Mw of PEAs, as well as the delivery mechanisms. This underlines the difficulty in achieving high efficiency and low toxicity with any type of polymers for specific cargo delivery. Nevertheless, the versatility of PEA series as delivery vectors for both pDNA and different $\mathrm{AO}$ chemistries affirms the potential of these polymers for a broad spectrum of applications of therapeutic agents.

\section{Acknowledgment}

The authors thank Mrs. Daisy M. Ridings and Ben Wagner with the Electron Microscopy Core Laboratory for the negative staining and TEM electron micrographs; Dr. David M. Foureau and Dr. Fei Guo for their technical assistance in FACS analysis. The authors gratefully acknowledge the financial support by the Carolinas Muscular Dystrophy Research Endowment at the Carolinas HealthCare Foundation and Carolinas Medical Center, Charlotte, NC.

The authors declare no conflicts of interest in relation to this paper.

\section{References}

1. Wagner KR, Lechtzin N, Judge DP (2007) Current treatment of adult Duchenne muscular dystrophy. Biochim Biophys Acta 1772: 229-237.

2. Foster K, Foster H, Dickson JG (2006) Gene therapy progress and prospects: Duchenne muscular dystrophy. Gene Ther 13: 1677-1685.

3. Järver P, O'Donovan L, Gait MJ (2014) A Chemical View of Oligonucleotides for Exon Skipping and Related Drug Applications. Nucleic Acid Ther 24: 37-47.

4. Koo T, Wood MJ (2013) Clinical trials using antisense oligonucleotides in Duchenne muscular dystrophy. Hum Gene Ther 24: 479-488.

5. Hoffman EP, Brown RH, Kunkel LM (1987) Dystrophin: the protein product of the Duchenne muscular dystrophy locus. Cell 51: 919-928.

6. Koenig M, Beggs $A H$, Moyer M (1989) The molecular basis for Duchenne versus Becker muscular dystrophy: correlation of severity with type of deletion. Am J Hum Genet 45: 498-506.

7. Wu B, Moulton HM, Iversen PL, Jiang J, Li J, et al. (2008) Effective rescue of dystrophin improves cardiac function in dystrophin-deficient mice by a modified morpholino oligomer. Proc Natl Acad Sci USA 105: 14814-14819.

8. Yokota, Lu QL, Partridge T, Masanori K, Akinori N, et al. (2009) Efficacy of systemic morpholino exon-skipping in duchenne dystrophy dogs. Annals of Neurology 65: 667-676.

9. Yin H, Moulton HM, Seow Y, Boyd C, Boutiller J, et al. (2008) Cell-penetrating peptide-conjugated antisense oligonucleotides restore systemic muscle and cardiac dystrophin expression and function. Hum Mol Genet 17: 3909-3918.

10. Kinali M, Arechavala-Gomeza V, Feng L, Cirak S, Hunt D, et al. (2009) Local restoration of dystrophin expression with the morpholino oligomer AVI-4658 in Duchenne muscular dystrophy: a single-blind, placebo-controlled, doseescalation, proof-of-concept study. The Lancet Neurology 8: 918-928.

11. Wu B, Cloer C, Shaban M, Moulton H, Lu P, et al. (2012) Long-term rescue of dystrophin expression and improvement in muscle pathology and function in dystrophic mdx mice by peptide-conjugated morpholino. Am J Pathol 181 : 392-400.

12. Yokota T, Hoffman E, Takeda S (2011) Antisense oligomediated multiple exon skipping in a dog model of duchenne muscular dystrophy. Methods in Molecular Biology 709: 299-312.

13. Wu B, Lu P, Benrashid E, Wu B, Lu P, Benrashid E, Malik S, Ashar J, et al. (2009) Dose-dependent restoration of dystrophin expression in cardiac muscle of dystrophic mice by systemically delivered morpholino. Gene Ther 17: 132-140.

14. Cirak S, Arechavala-Gomeza V, Guglieri M, Feng L, Torelli S, et al. (2011) Exon skipping and dystrophin restoration in patients with Duchenne muscular dystrophy after systemic phosphorodiamidate morpholino oligomer treatment An open-label, phase 2, dose-escalation study. Lancet 378: 595-605.

15. Wu B, Li Y, Morcos PA, Doran TJ, Lu P, et al. (2009) Octa-guanidine morpholino restores dystrophin expression in cardiac and skeletal muscles and ameliorates pathology in dystrophic mdx mice. Mol Ther 17: 864-871.

16. Aoki Y, Yokota T, Nagata T, Nakamura A, Tanihata J, et al. (2012) Bodywide skipping of exons $45-55$ in dystrophic mdx52 mice by systemic antisense delivery. Proc Natl Acad Sci USA 109: 13763-13768.

17. Lu QL, Rabinowitz A, Chen YC, Yokota H, Alter J, et al. (2005) Systemic delivery of antisense oligoribonucleotide restorers dystrophin expression in body-wide skeletal muscles. Proc Natl Acad Sci USA 102: 198-203.

18. van Deutekom JC, Janson AA, Ginjaar IB, Frankhuizen WS, et al. (2007) Local dystrophin restoration with antisense oligonucleotide PRO051. N Engl J Med 357: $2677-2686$ 
Citation: Wang M, Wu B, Tucker JD, Lu P, Lu Q (2015) Tris[2-(Acryloyloxy)Ethyl]lsocyanurate Cross-Linked Polyethylenimine Enhanced ExonSkipping of Antisense 2'-Omethyl Phosphorothioate Oligonucleotide in vitro and in vivo. J Nanomed Nanotechnol 6: 261. doi:10.4172/21577439.1000261

19. Goemans NM, Tulinius M, vanden Akker JT, et al. (2011) Systemic administration of PRO051 in Duchenne's muscular dystrophy. N Engl J Med 364: 1513-1522.

20. Arechavala-Gomeza V, Graham IR, Popplewell LJ (2007) Comparative analysis of antisense oligonucleotide sequences for targeted skipping of exon 51 during dystrophin pre-mRNA splicing in human muscle. Hum Gene Ther 18: 798-810.

21. Heemskerk $H$, De Winter $C$, van Kuik $P$, Heuvelmans $N$, Sabatelli $P$, et al. (2010) Preclinical PK and PD studies on 2'-O-methyl-phosphorothioate RNA antisense oligonucleotides in the mdx mouse model. Mol Ther 18: 1210-1217.

22. Takeshima $Y$, Yagi M, Wada H, Matsuo M (2005) Intraperitoneal administration of phosphorothioate antisense oligodeoxynucleotide against splicing enhancer sequence induced exon skipping in dystrophin mRNA expressed in $\mathrm{mdx}$ skeletal muscle. Brain \& Development 27: 488-493.

23. Tanganyika-de Winter CL, Heemskerk H, Karnaoukh TG, Putten MV, Kimpe SJ, et al. (2012) Long-term exon skipping studies with 2'-O-methyl phosphorothioate antisense oligonucleotides in dystrophic mouse models. Mol Ther Nucl Acids 1: e43.

24. Sirsi SR, Schray RC, Guan X, Lykens NM, Williams HJ, et al. (2008) Functionalized PEG-PEI copolymers complexed to exon-skipping oligonucleotides improve dystrophin expression in mdx mice. Hum Gene Ther 19: $795-806$.

25. Williams JH, Sirsi SR, Latta DR, Lutz GJ (2006) Induction of dystrophin expression by exon skipping in mdx mice following intramuscular injection of antisense oligonucleotides complexed with PEG-PEI copolymers. Mol Ther 14: 88-96.
26. Wang M, Wu B, Lu P, Cloer C, Tucker JD, et al. (2013) Polyethylenimine modified pluronics (PCMs) improve morpholino oligomer delivery in cell culture and dystrophic mdx mice. Mol Ther 21: 210-216.

27. Wang M, Wu B, Lu P, Tucker JD, Milazi S, et al. (2014) Pluronic-PEI Copolymers Enhance Exon-skipping of 2'-O-Methyl Phosphorothioate Oligonucleotide in Cell Culture and Dystrophic mdx Mice. Gene Ther 21: 52-59.

28. Wang M, Tucker JD, Lu P, Wu B, Cloer C, et al. (2012) Tris[2-(acryloyloxy) ethyl]isocyanurate cross-linked low-molecular-weight polyethylenimine as gene delivery carriers in cell culture and dystrophic $\mathrm{mdx}$ mice. Bioconjug Chem 23: 837-845.

29. Wang M, Wu B, Tucker JD, Cloer C, Lu QL (2014) Evaluation of tris[2(acryloyloxy)ethyl]isocyanurate cross-linked polyethylenimine as antisense morpholino oligomer delivery vehicle in cell culture and dystrophic mdx mice. Hum Gene Ther 25: 419-427.

30. Arote RB, Lee ES, Jiang HL, Kim YK, Choi YJ, et al. (2009) Efficient gene delivery with osmotically active and hyperbranched poly(ester amine)s. Bioconjugate Chem 20 : 2231-2241.

31. Zhu L, Mahato RM (2010) Lipid and polymeric carrier-mediated nucleic acid delivery. Expert Opin Drug Deliv 7: 1209-1226.

32. Ruponen M, Ronkko S, Honkakoski P, Pelkonen J, Tammi M, et al. (2001) Extracellular glycosaminoglycans modify cellular trafficking of lipoplexes. J Bio Chem 276: 33875-33880. 